\title{
HIDRÓXIDO DE NÍQUEL SUPORTADO EM CARBONO: UM CATALISADOR DE BAIXO CUSTO PARA A ELETRO-OXIDAÇÃO DE ALCOÓIS EM MEIO ALCALINO ${ }^{*}$
}

\author{
Marcelo Rodrigues da Silva e Antonio Carlos Dias Ângelo \\ Departamento de Química, Faculdade de Ciências, Universidade Estadual Paulista, CP 473, 17033-360 Bauru - SP, Brasil \\ Luiz Henrrique Dall'Antonia* \\ Departamento de Química, Centro de Ciências Exatas, Universidade Estadual de Londrina, CP 6001, 86051-580 Londrina - PR, Brasil
}

Recebido em 5/4/10; aceito em 6/10/10; publicado na web em 3/11/10

\begin{abstract}
CARBON SUPPORTED NICKEL HYDROXIDE: A LOW COST CATALYST FOR ALCOHOL ELECTRO-OXIDATION IN ALKALINE MEDIUM. The present manuscript shows the synthesis of nickel hydroxide supported in carbon $\left(\mathrm{Ni}(\mathrm{OH})_{2} / \mathrm{C}\right)$ as a alternative material for catalytic alcohol oxidation in alkaline medium. The $\mathrm{Ni}(\mathrm{OH})_{2} / \mathrm{C}$ was synthesized in different percentage using a sonic bath. No current densities variation during successive cyclic voltammetry experiments was observed. The $\mathrm{Ni}(\mathrm{OH})_{2} / \mathrm{C}$ electrodes exhibit a potent and persistent electrocatalytic activity towards the oxidation of different alcohols. In addition, alcohols electooxidation occurs in less positive potential compared with noble metal catalyst.
\end{abstract}

Keywords: nickel hydroxide; alcohols; electro-oxidation.

\section{INTRODUÇÃO}

Nas últimas décadas, grandes esforços têm sido realizados para a utilização de combustíveis líquidos em células a combustível de baixa temperatura de operação. ${ }^{1}$ Alcoóis de baixo peso molecular como, por exemplo, metanol, etanol e etileno glicol se apresentam como combustíveis promissores, pois apresentam baixo custo de obtenção e são de fácil estocagem. Além disso, apresentam elevadas densidades teóricas de energia, 6,1; 8 e 5,2 $\mathrm{kWh} \mathrm{kg}^{-1}$ para metanol, etanol e etileno glicol, respectivamente. ${ }^{2}$

A molécula de metanol, que apresenta apenas um átomo de carbono, é o álcool mais fácil de ser oxidado, formando como principal intermediário de reação o monóxido de carbono. Esta espécie intermediária apresenta forte interação com a platina, adsorvendo-se quimicamente em seus sítios ativos na forma linear ou em ponte, bloqueando a superfície eletródica e, consequentemente, diminuindo a eficiência do catalisador. ${ }^{3}$ Considerando a molécula de etanol, a quebra da ligação C-C é muito difícil de acontecer à baixa temperatura, gerando acetaldeído e acido acético/acetato como principais intermediários de reação, o que leva a uma baixa eficiência faradaica (17-33\% da energia teórica). ${ }^{2}$ A quebra da ligação C-C da molécula do etileno glicol também é muito difícil de ocorrer a baixas temperaturas. Alguns trabalhos reportados na literatura sugerem que a molécula de etileno glicol é oxidada a espécies do tipo oxalato ( $\left.\mathrm{COO}^{-}-\mathrm{COO}^{-}\right)$sendo que, em seguida, 8 mols de elétrons são trocados por mol de etileno glicol em vez de 10 mols de elétrons para completar a oxidação a $\mathrm{CO}_{2}$, o que implica em uma eficiência faradaica de $80 \% .^{4-7}$

O maior desafio para utilização destes alcoóis em sistemas reais de células a combustível é a busca por catalisadores que apresentam elevada eletroatividade frente à reação de oxidação dos mesmos. Ligas metálicas à base de platina ainda são os catalisadores mais utilizados atualmente. ${ }^{8,9}$ Com a utilização destas ligas, a susceptibilidade dos sítios de platina pelo intermediário CO é diminuída, pois o segundo metal ligado à platina pode promover a formação de espécies oxige-

*e-mail: luizh@uel.br

"Artigo em homenagem ao Prof. Hans Viertler nadas a baixos potenciais, onde estas espécies oxigenadas oxidam o $\mathrm{CO}_{\text {ads }}$ a $\mathrm{CO}_{2}$ através do mecanismo bifuncional. ${ }^{10}$ Apesar das ligas metálicas à base de platina serem excelentes eletrocatalisadores para a oxidação de alcoóis, as mesmas não apresentam estabilidade físicoquímica dependendo do intervalo de potencial aplicado, além do fato de que a platina é um metal nobre e, consequentemente, encarece a célula a combustível como um todo, dificultando a inserção destes dispositivos no mercado consumidor.

Outra questão a ser considerada para utilização de determinados catalisadores frente à oxidação de alcoóis é a escolha do eletrólito a ser utilizado. Em eletrólito alcalino, a cinética das reações eletródicas é favorecida, pois as espécies $\mathrm{OH}^{-}$livres em solução diminuem a susceptibilidade ao envenenamento do catalisador, o que não acontece em eletrólito ácido. ${ }^{11}$ Outra vantagem do eletrólito alcalino é a possibilidade de utilização de metais não nobres como catalisadores, por exemplo, $\mathrm{Ni}$ e $\mathrm{NiCu} .{ }^{12,13}$

Neste contexto, a busca por materiais alternativos que apresentem elevada eficiência faradaica e baixos custos de obtenção para atuarem como catalisador frente à eletro-oxidação de alcoóis se faz necessária. Um material que tem sido bastante estudado nos últimos anos é o hidróxido de níquel. São várias as aplicações tecnológicas deste material. Usualmente é empregado como material ativo para eletrodos positivos de níquel, dispositivos eletrocrômicos, promotor catalítico para reações que envolvem evolução de oxigênio e, recentemente, tem sido reportado como sensor eletroquímico para detecção de uréia e glicina. ${ }^{14-19}$ Duas vias de obtenção deste hidróxido são amplamente empregadas, a primeira por eletrodeposição catódica, ${ }^{14}$ produzindo um filme de espessura controlada, e a segunda utilizando o método sonoquímico, ${ }^{20}$ produzindo nanoestruturas. Neste contexto, o hidróxido de níquel surge como potencial material de eletrodo a ser utilizado como eletrocatalisador. Alguns trabalhos reportam o uso deste hidróxido como catalisador para eletro-oxidação de alcoóis de baixo peso molecular. ${ }^{21-25}$ Amjad e colaboradores estudaram a eletro-oxidação de fenil etanol, benzil e alil álcool sob um filme eletroativo de hidróxido de níquel formado sob a superfície de um eletrodo de níquel metálico, através da técnica de voltametria cíclica, demonstrando que o estado oxidado do hidróxido promove a oxidação 
dos alcoóis. ${ }^{26}$ Além disso, os autores sugerem que o ácido carboxílico é o único produto formado a partir da eletro-oxidação do fenil etanol e alil álcool e que uma mistura de aldeído/ácido carboxílico é encontrada como produto a partir da eletro-oxidação do benzil álcool. El-Shafei estudou a eletroatividade do hidróxido de níquel depositado sobre eletrodo de carbono vítreo frente à eletro-oxidação do metanol, evidenciando a excelente eletroatividade do material e encontrando, também, que o estado oxidado do hidróxido proporciona a eletro-oxidação da molécula de metanol..$^{27}$ Jafarian e colaboradores estudaram a eletro-oxidação do metanol sob filme de hidróxido de níquel formado galvanostaticamente sobre eletrodo de níquel metálico e um filme de hidróxido de níquel dopado com cobre formado galvanostaticamente sobre eletrodo de $\mathrm{Ni}-\mathrm{Cu}$, que indicaram ser mais eletroativos que o hidróxido depositado sobre níquel. ${ }^{28}$

Diante dos promissores resultados encontrados na literatura envolvendo o hidróxido de níquel frente à eletro-oxidação de alcoóis, principalmente metanol, observa-se a necessidade de novas rotas sistemáticas para obtenção deste material de eletrodo. Eletrocatalisadores suportados são uma ótima alternativa, uma vez que para aplicações tecnológicas em sistemas reais de células a combustíveis de baixa temperatura de operação, especificamente no compartimento anódico, há a necessidade da difusão facilitada dos combustíveis líquidos pela camada catalítica. ${ }^{29-31}$

O presente trabalho descreve a síntese do hidróxido de níquel com baixíssimo custo de obtenção, adaptado do método sonoquímico, ${ }^{20} \mathrm{com}$ elevada área superficial, suportado em carbono. Baseados na potencialidade tecnológica deste hidróxido servir como material de eletrodo foram avaliadas propriedades eletrocatalíticas frente à eletro-oxidação em meio alcalino dos alcoóis: metanol, etanol e etileno glicol. ${ }^{21-26}$

\section{PARTE EXPERIMENTAL}

\section{Síntese do catalisador $\mathrm{Ni}(\mathrm{OH})_{2} / \mathrm{C}$}

O hidróxido de níquel suportado em carbono $\left(\mathrm{Ni}(\mathrm{OH})_{2} / \mathrm{C}\right)$ foi sintetizado em diferentes proporções em massa de metal/suporte $(30: 70 ; 50: 50 ; 70: 30)$. Os reagentes utilizados foram $\mathrm{Ni}\left(\mathrm{NO}_{3}\right)_{2} \cdot 6 \mathrm{H}_{2} \mathrm{O}$ (Sigma-Aldrich), $\mathrm{NH}_{4} \mathrm{OH}$ (Merck, P.A.) e carbono Vulcan (Cabot, $\mathrm{XC}-72$ ). Em um erlenmeyer com capacidade para $250 \mathrm{~mL}$, foram colocados $100 \mathrm{~mL}$ de uma solução de $\mathrm{Ni}\left(\mathrm{NO}_{3}\right)_{2} \cdot 6 \mathrm{H}_{2} \mathrm{O}$, mantendo sempre as proporções desejadas, ou seja, de 30, 50 e $70 \%$ em massa de níquel e 70, 50 e $30 \%$ de carbono Vulcan, respectivamente. Em seguida, $2 \mathrm{~mL}$ de $\mathrm{NH}_{4} \mathrm{OH}_{\text {(conc.) }}$ foram adicionados e manteve-se o sistema em banho de ultrassom por $1 \mathrm{~h}$. O banho de ultrassom utilizado foi da marca Limp Sonico Embrassel Tec (40 Hz e $35 \mathrm{~W}$ ) sendo que este apresentou boa estabilidade, sem bruscas variações de potência, durante todo o experimento. $\mathrm{O}$ volume de $\mathrm{NH}_{4} \mathrm{OH}_{\text {(conc.) }}$ em excesso provoca a precipitação de todo $\mathrm{Ni}^{2+}$ livre em solução na forma de $\mathrm{Ni}(\mathrm{OH})_{2}$, uma vez que seu $\mathrm{K}_{\mathrm{PS}}$ é igual a 5,46 x 10 $0^{-16}$. Ao final da síntese, o produto foi lavado por diversas vezes com água deionizada, separado por centrifugação e seco em um dessecador por $24 \mathrm{~h}$.

\section{Caracterização física}

O material eletródico sintetizado foi caracterizado por difração de raios-x (DRX), método do pó, através de um equipamento da Rigaku Rint 2000 Diffractometer com fonte de radiação $\mathrm{Cu}-\mathrm{K} \alpha_{1}(\lambda=1,5406$ $\AA$ ). Os difratogramas foram coletados com uma média de 3 varreduras sucessivas a uma velocidade de $1 \%$ min, nos intervalos de $2 \theta=10-80^{\circ}$.

\section{Caracterização e avaliação eletroquímica}

A caracterização e avaliação eletroquímica do $\mathrm{Ni}(\mathrm{OH})_{2} / \mathrm{C}$ foram realizadas por voltametria cíclica e cronoamperometria. Uma suspen- são contendo o $\mathrm{Ni}(\mathrm{OH})_{2} / \mathrm{C}$ foi preparada utilizando-se o pó catalítico, $200 \mu \mathrm{L}$ de Nafion ${ }^{\circledR}$ (5,5\% p), $200 \mu \mathrm{L}$ de álcool isopropílico (Merck, p.a.) e $100 \mu \mathrm{L}$ de água deionizada, dispersando a mesma em ultrassom por $30 \mathrm{~min}$. Uma alíquota de $10 \mu \mathrm{L}$ desta suspensão foi aplicada sobre a superfície do eletrodo de carbono vítreo (área geométrica de $0,031 \mathrm{~cm}^{2}$ ) e seca em um dessecador por $24 \mathrm{~h}$. O eletrodo de carbono vítreo com a camada fina de Pt/C foi preparado da mesma maneira, com a mesma quantidade de platina na solução, para que ambos os eletrodos tivessem a mesma quantidade de metal na camada fina. Diante disso, os parâmetros eletroquímicos do $\mathrm{Ni}(\mathrm{OH})_{2} / \mathrm{C}$ frente à eletro-oxidação dos combustíveis orgânicos serão comparados com os parâmetros eletroquímicos da $\mathrm{Pt} / \mathrm{C}$. As medidas eletroquímicas foram realizadas em temperatura ambiente, utilizando-se uma solução 0,1 mol L-1 de KOH (Merck, p.a.) como eletrólito suporte e soluções de metanol, etanol e etileno glicol 0,1 $\mathrm{mol} \mathrm{L}^{-1}$ (Merck, p.a.) em meio $\mathrm{KOH}$. Os dados foram obtidos por intermédio de um potenciostato/ galvanostato EG\&G PAR283, controlado pelo software M270. Um eletrodo de $\mathrm{Ag} / \mathrm{AgCl}\left(3 \mathrm{~mol} \mathrm{~L}{ }^{-1}, \mathrm{KCl}\right)$ foi usado como eletrodo de referência e um fio de platina (com $10 \mathrm{~cm}$ de comprimento e $0,5 \mathrm{~mm}$ de diâmetro) como contra eletrodo.

\section{RESULTADOS E DISCUSSÃO}

Uma vez que foram sintetizados os materiais eletródicos, contendo hidróxido de níquel suportado, em diferentes proporções de metal/ carbono, uma análise e caracterização dos mesmos se faz necessária. A Figura 1 apresenta os perfis de difração de raios-x para as diferentes amostras obtidas. A partir dos resultados alcançados observa-se que ambas as proporções utilizadas produzem materiais que apresentaram perfis de difração característicos de materiais cristalinos, onde os planos indexados confirmam a estrutura cristalina deste material como sendo a fase $\beta-\mathrm{Ni}(\mathrm{OH})_{2}$, pertencente ao sistema hexagonal (P3m1 (164)). Um pico de difração em aproximadamente $2 \theta=25^{\circ}$ é atribuído ao suporte carbono Vulcan. ${ }^{32}$ Outro pico de difração de baixa intensidade aparece em aproximadamente $2 \theta=16,8^{\circ}$ e pode ser atribuído a alguma impureza presente no suporte carbono Vulcan. ${ }^{33}$

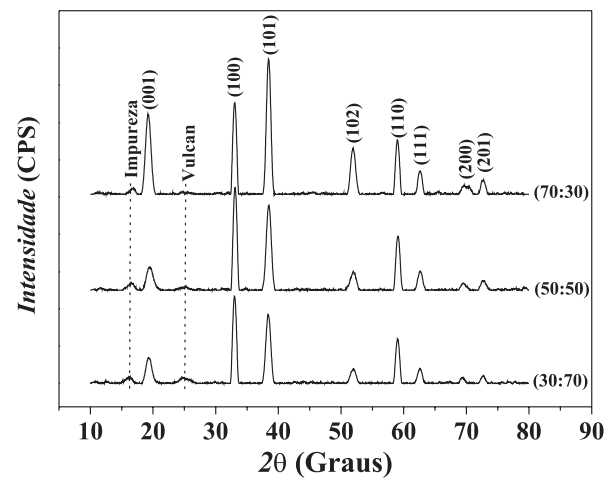

Figura 1. Difratograma do $\mathrm{Ni}(\mathrm{OH})_{2} / \mathrm{C}$ sintetizado em diferentes proporções em massa de metal/carbono

A Tabela 1 ilustra os parâmetros de rede do $\mathrm{Ni}(\mathrm{OH})_{2} / \mathrm{C}$ calculados através das Equações 1 e 2, assim como uma estimativa do tamanho médio de partícula, obtido através da Equação de Scherrer. ${ }^{34}$ Comparando os parâmetros de rede calculados pelas Equações 1 e 2 com os valores teóricos $a=3,13 \AA$ e $c=4,61 \AA$, $^{32}$ observa-se que não ocorreram variações significativas, encontrando-se um erro menor que $1 \%$.

$$
a=\frac{\lambda}{\sqrt{3} \operatorname{sen} \theta} \sqrt{h^{2}+h k+k^{2}}
$$


Tabela 1. Tamanho de partícula e parâmetros de rede do $\mathrm{Ni}(\mathrm{OH})_{2} / \mathrm{C}$ em diferentes proporções em metal/carbono

\begin{tabular}{lccc}
\hline $\mathrm{Ni}(\mathrm{OH})_{2} / \mathrm{C}$ & $\begin{array}{c}\text { Tamanho de } \\
\text { partícula }(\mathrm{nm})\end{array}$ & \multicolumn{2}{c}{ Parâmetros de rede $(\AA)$} \\
$\mathrm{a}$ & $\mathrm{c}$ \\
\hline $30: 70$ & 8,7 & 3,133 & 4,593 \\
$50: 50$ & 8,7 & 3,136 & 4,560 \\
$70: 30$ & 9,6 & 3.133 & 4,626 \\
\hline
\end{tabular}

$$
c=\frac{\lambda}{2 \operatorname{sen} \theta} l
$$

A caracterização eletroquímica do $\mathrm{Ni}(\mathrm{OH})_{2} / \mathrm{C}$ foi realizada por voltametria cíclica. O perfil voltamétrico do hidróxido sintetizado em diferentes proporções está ilustrado na Figura 2. A partir dos dados obtidos é possível observar dois picos no perfil de corrente em função do potencial em aproximadamente 0,25 e $0,5 \mathrm{~V}$, correspondendo ao par redox $\mathrm{Ni}^{2+} / \mathrm{Ni}^{3+}$, o estado reduzido e oxidado do hidróxido de níquel. De acordo com os dados obtidos é possível notar que o material com a proporção de 30:70 $\left(\mathrm{Ni}(\mathrm{OH})_{2}: \mathrm{C}\right)$ apresentou maior densidade de corrente em comparação ao material com 70:30 (Ni(OH) $\left.)_{2}: \mathrm{C}\right)$. Neste caso, acredita-se que no material sintetizado com uma menor proporção de metal, o respectivo hidróxido precipita de forma mais dispersa sobre o suporte e, consequentemente, apresentaria uma maior área ativa.

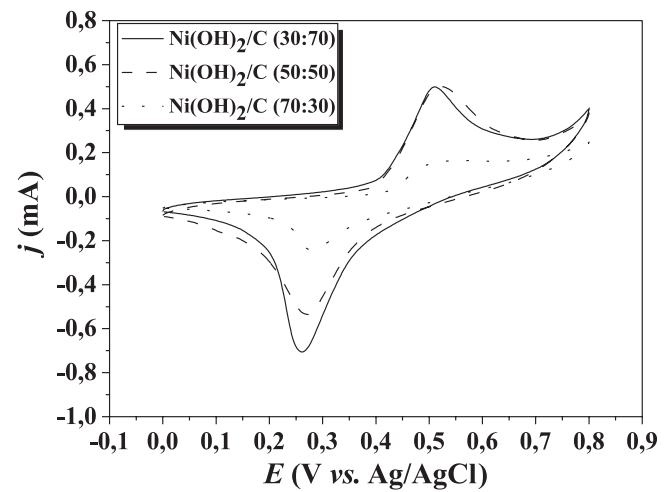

Figura 2. Perfil voltamétrico do $\mathrm{Ni}(\mathrm{OH})_{2} / \mathrm{C} \mathrm{em} 0,1 \mathrm{~m} \mathrm{~L}^{-1}$ de KOH. $d E / d t=$ $50 \mathrm{mVs}^{-1}$

A fim de verificar a estabilidade eletroquímica do $\mathrm{Ni}(\mathrm{OH})_{2} / \mathrm{C}$, o respectivo material, na proporção metal/carbono que apresentou maior densidade de corrente $\left(30: 70\left(\mathrm{Ni}(\mathrm{OH})_{2}: \mathrm{C}\right)\right)$, foi submetido a sucessivas ciclagens de potencial entre $0-0,8 \mathrm{~V}$. Os resultados, mostrados na Figura 3, indicam que o material em questão não apresentou variação acentuada de corrente na varredura anódica, transição $\mathrm{Ni}^{2+} /$ $\mathrm{Ni}^{3+}$ e na varredura catódica, transição $\mathrm{Ni}^{3+} / \mathrm{Ni}^{2+}$, confirmando sua estabilidade eletroquímica.

A atividade eletrocatalítica do $\mathrm{Ni}(\mathrm{OH})_{2} / \mathrm{C}(30: 70)$, avaliada por voltametria cíclica, frente à oxidação dos alcoóis está ilustrada na Figura 4a. Através dos voltamogramas, observa-se que o respectivo hidróxido apresenta excelente eletroatividade. A platina comercial da E-Tek (Pt/C) foi usada como parâmetro de comparação. A camada catalítica dos eletrodos de carbono vítreo contendo os filmes de $\mathrm{Ni}(\mathrm{OH})_{2} / \mathrm{C}$ e Pt/C foi preparada de modo que apresentasse a mesma quantidade de metal, para posterior comparação dos parâmetros eletroquímicos. A Figura 4b ilustra os perfis voltamétricos da $\mathrm{Pt} / \mathrm{C}$ frente à oxidação dos alcoóis. Os parâmetros eletroquímicos utilizados para avaliar a eletroatividade do $\mathrm{Ni}(\mathrm{OH})_{2} / \mathrm{C}$ em relação a $\mathrm{Pt} / \mathrm{C}$ foram onset potential $(O P)$, densidade de corrente máxima de oxidação

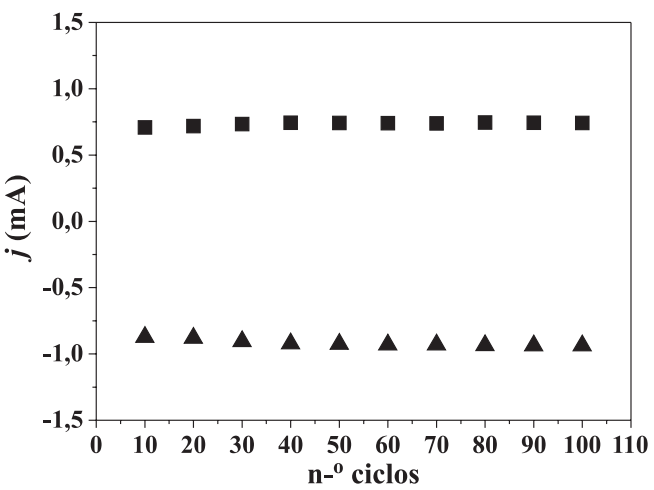

Figura 3. Estabilidade eletroquímica do $\mathrm{Ni}(\mathrm{OH})_{2} / \mathrm{C}$ (30:70) obtida por voltametria cíclica em $\mathrm{KOH} 0,15 \mathrm{~mol} \mathrm{~L}^{-1}$. Valores de corrente de pico nas varreduras: $(\mathbf{\square})$ anódica e $(\mathbf{\Delta})$ catódica. $d E / d t=10 \mathrm{mV} \mathrm{s}^{-1}$
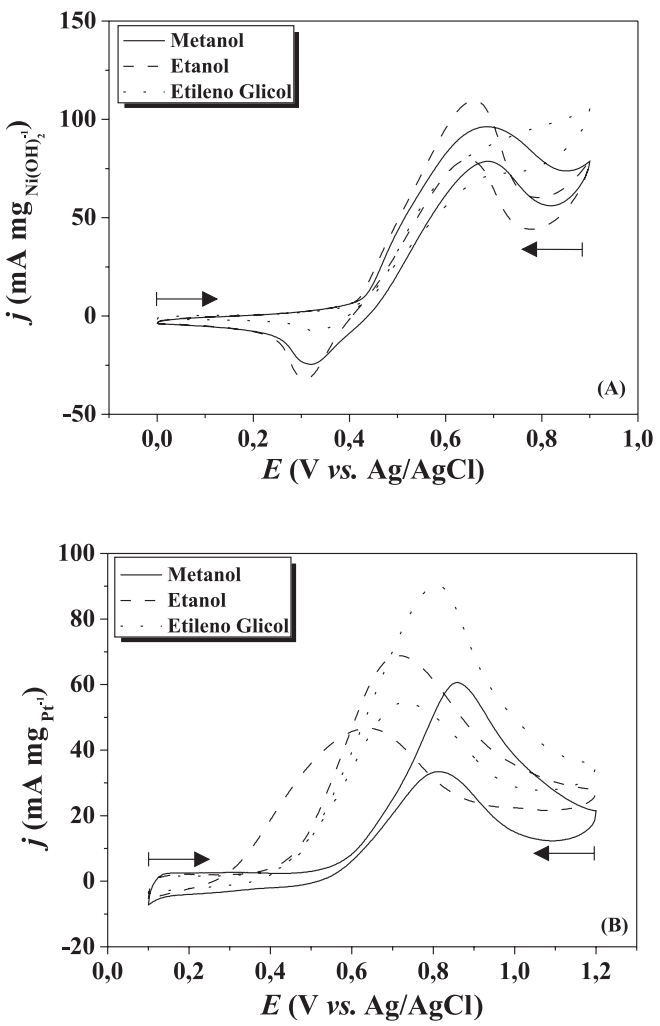

Figura 4. Voltamogramas cíclicos do (a) $\mathrm{Ni}(\mathrm{OH})_{2} / \mathrm{C}(30: 70)$ e (b) Pt/C frente à oxidação dos alcoóis em $\mathrm{KOH} 0,1 \mathrm{~mol} \mathrm{~L}^{-1}$. dE/dt $=20 \mathrm{mV} \mathrm{s}^{-1}$

(j) e potencial de pico obtido na densidade de corrente máxima de oxidação $(E)$. O termo $O P$ é definido como sendo o potencial onde se iniciam os processos faradaicos.

Um mecanismo geral para a oxidação de alcoóis primários foi proposto por Fleishmann e colaboradores: $:^{35,36}$

$$
\begin{gathered}
\mathrm{Ni}(\mathrm{OH})_{2}+\mathrm{OH}^{-} \rightarrow \mathrm{NiOOH}+\mathrm{H}_{2} \mathrm{O}+\mathrm{e}^{-} \\
\mathrm{NiOOH}+\mathrm{RCH}_{2} \mathrm{OH} \rightarrow \mathrm{Ni}(\mathrm{OH})_{2}+\mathrm{RCHOH} \\
\mathrm{RCHOH}+3 \mathrm{OH}^{-} \rightarrow \mathrm{RCOOH}+2 \mathrm{H}_{2} \mathrm{O}+3 \mathrm{e}^{-}
\end{gathered}
$$

onde, a etapa 3 corresponde à oxidação superficial de $\mathrm{Ni}(\mathrm{OH})_{2}$ para NiOOH. A etapa 4 é a reação superficial que envolve a adsorção do álcool na superfície do $\mathrm{NiOOH}$ com a subsequente remoção do hidrogênio do álcool pelo $\mathrm{NiOOH}$. A camada de $\mathrm{Ni}(\mathrm{OH})_{2}$ formada 
Tabela 2. Parâmetros eletroquímicos $\left(j, O P\right.$ e $\left.E_{j \max }\right)$ obtidos por voltametria cíclica frente à oxidação dos alcoóis em $\mathrm{KOH} 0,1 \mathrm{~mol} \mathrm{\textrm {L } ^ { - 1 }}$. dE/dt $=20 \mathrm{mV} \mathrm{s}{ }^{-1}$

\begin{tabular}{lcccccccccc}
\hline Catalisadores & \multicolumn{9}{c}{ Combustíveis } \\
& \multicolumn{3}{c}{ Metanol } & \multicolumn{3}{c}{ Etanol } & \multicolumn{3}{c}{ Etileno Glicol } \\
\hline & $j\left(\mathrm{~mA} \mathrm{mg}^{-1}\right)$ & $O P(\mathrm{~V})$ & $E_{j_{\max }}(\mathrm{V})$ & $j\left(\mathrm{~mA} \mathrm{mg}^{-1}\right)$ & $O P(\mathrm{~V})$ & $E_{j_{\max }}(\mathrm{V})$ & $j\left(\mathrm{~mA} \mathrm{mg}^{-1}\right)$ & $O P(\mathrm{~V})$ & $E_{j_{\max }}(\mathrm{V})$ \\
\hline $\mathrm{Pt} / \mathrm{C}$ & 60 & 0,54 & 0,86 & 68 & 0,40 & 0,71 & 90 & 0,41 & 0,80 \\
$\mathrm{Ni}(\mathrm{OH})_{2} / \mathrm{C}$ & 96 & 0,41 & 0,69 & 110 & 0,40 & 0,61 & 82 & 0,40 & 0,63 \\
\hline
\end{tabular}

nesta mesma etapa é prontamente reoxidada a oxi-hidróxido devido ao potencial bastante positivo aplicado, regenerando (etapa 3 ) proporcionando novamente o processo de oxidação. Finalmente, a etapa 5 ilustra a subsequente e rápida oxidação do intermediário formando o correspondente ácido carboxílico. A formação de aldeídos e/ou ácidos carboxílicos sobre superfícies de hidróxido de níquel foi observada e relatada na literatura, corroborando o mecanismo proposto. ${ }^{37-40}$

Com relação ao combustível metanol, o $\mathrm{Ni}(\mathrm{OH})_{2} / \mathrm{C}$ apresentou eletroatividade superior a $\mathrm{Pt} / \mathrm{C}$ em todos os parâmetros eletroquímicos analisados, confirmando a potencialidade catalítica deste material. A densidade máxima de corrente obtida pelo hidróxido foi de 96 $\mathrm{mA} \mathrm{mg}{ }^{-1}$ em relação a $60 \mathrm{~mA} \mathrm{mg} \mathrm{mg}^{-1} \mathrm{da} \mathrm{Pt} / \mathrm{C}$; os resultados estão sumarizados na Tabela 2. Os processos de transferência eletrônica na superfície do $\mathrm{Ni}(\mathrm{OH})_{2} / \mathrm{C}$ iniciam-se em baixos potenciais, como evidenciado pelo valor de $O P, 0,41 \mathrm{~V}$, em relação a $0,54 \mathrm{~V}$ da $\mathrm{Pt} / \mathrm{C}$. Em termos de potencial de corrente máxima de oxidação $\left(E_{j_{\max }}\right)$, o $\mathrm{Ni}(\mathrm{OH})_{2} / \mathrm{C}$ apresentou um deslocamento de $0,17 \mathrm{~V}$ para potenciais menos positivos. O melhor desempenho deste hidróxido em relação a $\mathrm{Pt} / \mathrm{C}$ pode estar associado ao excesso de espécies $\mathrm{OH}$ presentes na estrutura superficial do hidróxido, como proposto nas Equações 3 e 4.

Com relação ao combustível etanol, o $\mathrm{Ni}(\mathrm{OH})_{2} / \mathrm{C}$ também apresentou melhor eletroatividade em todos os parâmetros eletroquímicos analisados quando comparado a Pt/C (E-Tek). A densidade máxima de corrente obtida por este hidróxido foi de $110 \mathrm{~mA} \mathrm{mg}^{-1}$ contra $68 \mathrm{~mA} \mathrm{mg}{ }^{-1} \mathrm{da} \mathrm{Pt} / \mathrm{C}$ comercial, Tabela 2. Com relação ao $O P$, observam-se valores semelhantes para os dois catalisadores, indicando que a oxidação do etanol começa praticamente no mesmo potencial para ambos, em aproximadamente $0,40 \mathrm{~V}$. Em termos de potencial de corrente máxima de oxidação, o $\mathrm{Ni}(\mathrm{OH})_{2} / \mathrm{C}$ apresentou um deslocamento de $0,10 \mathrm{~V}$ para potenciais menos positivos.

Os parâmetros eletroquímicos frente à oxidação do etileno glicol mostraram que o $\mathrm{Ni}(\mathrm{OH})_{2} / \mathrm{C}$ apresentou uma menor densidade máxima de corrente $\left(82 \mathrm{~mA} \mathrm{mg}^{-1}\right)$ quando comparado a Pt/C $(90 \mathrm{~mA}$ $\mathrm{mg}^{-1}$ ). Porém, em termos de $O P$ o hidróxido suportado apresentou um potencial menos positivo, indicando que a oxidação do combustível se inicia em baixos potenciais. Além disso, um deslocamento de $0,17 \mathrm{~V}$ no potencial de densidade de corrente máxima ocorre com o $\mathrm{Ni}(\mathrm{OH})_{2} / \mathrm{C}$ na oxidação do etileno glicol em relação a Pt/C.

$\mathrm{O}$ desempenho eletroquímico do $\mathrm{Ni}(\mathrm{OH})_{2} / \mathrm{C}$ foi avaliado por cronoamperometria. A Figura 5a ilustra os perfis de decaimento das curvas amperométricas referentes a cada combustível com o potencial controlado em 0,50 V. Observa-se que em aproximadamente 50 s, o material em questão já atinge estabilidade eletroquímica frente à eletro-oxidação do metanol, etanol e etileno glicol, não apresentado variação de corrente até o tempo máximo de $600 \mathrm{~s}$. As curvas amperométricas da $\mathrm{Pt} / \mathrm{C}$ frente à eletro-oxidação dos alcoóis estão ilustradas na Figura 5b. Observa-se que a Pt/C adquire estabilidade eletroquímica apenas na oxidação do metanol, após aproximadamente 100 s. Com relação às curvas de decaimento cronoamperométrico do etanol e etileno glicol, o material em questão não adquire estabilidade, apresentando uma perda de corrente até o tempo máximo de $600 \mathrm{~s}$. Neste caso, acredita-se que esta perda de corrente é em função do bloqueio superficial da platina por espécies intermediárias como, por exemplo, o CO. ${ }^{10}$ As densidades de corrente obtidas através das curvas amperométricas para cada catalisador, referentes aos combustíveis metanol, etanol e etileno glicol, estão contidas na Tabela 3.
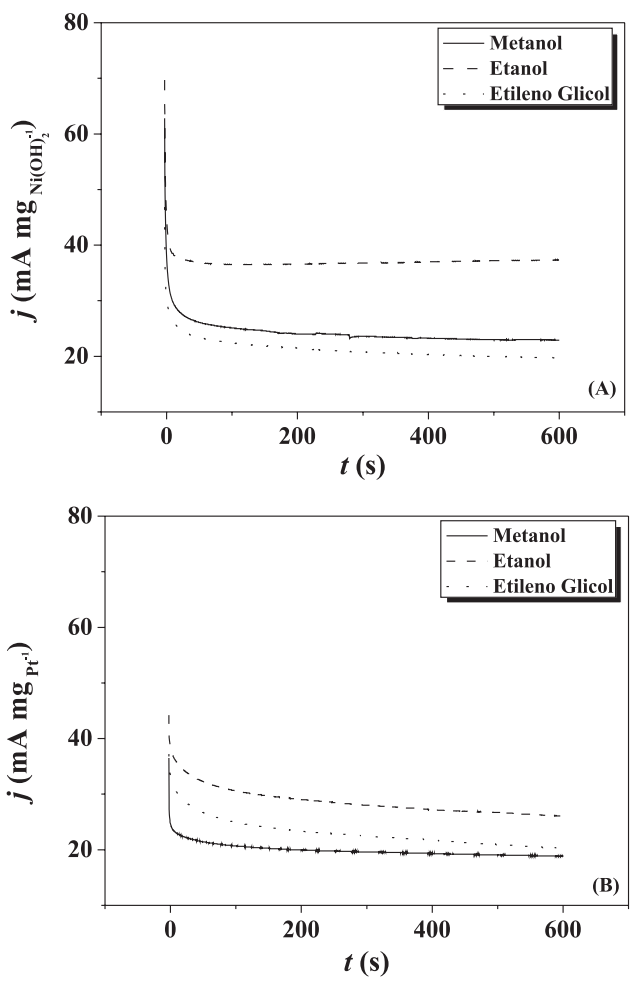

Figura 5. Curvas cronoamperométricas do (a) $\mathrm{Ni}(\mathrm{OH})_{2} / \mathrm{C}$ (30:70) e (b) $\mathrm{Pt} / \mathrm{C}$ frente à eletro-oxidação dos alcoóis em $500 \mathrm{mV}$

Através das densidades de corrente, observa-se que o $\mathrm{Ni}(\mathrm{OH})_{2} / \mathrm{C}$ mostrou ser o melhor catalisador para oxidação de metanol e etanol, apresentando 23 e $37 \mathrm{~mA} \mathrm{mg}^{-1}$, respectivamente, em relação a 18 e $26 \mathrm{~mA} \mathrm{mg}^{-1} \mathrm{da} \mathrm{Pt/C}$, respectivamente. Com relação à oxidação do etileno glicol, os dois catalisadores apresentaram resultados semelhantes, como já evidenciados por voltametria cíclica, Figura 4(a e b). A partir dos cronoamperogramas obtidos pode-se calcular a constante de velocidade para a catálise de oxidação dos diferentes alcoóis. A constante de velocidade para o processo catalítico estudado neste trabalho pode ser calculada a partir da Equação 6:41

$$
\frac{I_{c a t}}{I_{I .}}=\pi^{\frac{1}{2}}\left(k_{o b s} C_{0} t\right)^{\frac{1}{2}}
$$

onde $\mathrm{k}_{\mathrm{obs}}$ é a constante de velocidade observada para a catálise $\left(\mathrm{M}^{-1}\right.$ $\left.\mathrm{s}^{-1}\right), \mathrm{C}_{0}$ a concentração do álcool $(\mathrm{M})$, t o tempo do experimento (600 s) e $I_{\text {cat }}$ e $I_{L}$ são as correntes dos eletrodos na presença e na ausência do álcool, respectivamente. Os resultados obtidos estão apresentados na Tabela 3. O eletrodo modificado com o hidróxido de níquel suportado em carbono demonstrou maior valor da constante catalítica para todos os combustíveis, em comparação com a constante catalítica da $\mathrm{Pt} / \mathrm{C}$ (E-Tek). 
Tabela 3. Densidade de corrente (j) obtidas através das curvas cronoamperométricas em $600 \mathrm{~s}$

\begin{tabular}{lcccccc}
\hline Catalisadores & \multicolumn{5}{c}{$\begin{array}{c}\text { Combustíveis } \\
\text { Metanol }\end{array}$} & \multicolumn{2}{c}{ Etanol } & \multicolumn{2}{c}{ Etileno Glicol } \\
\hline & $\begin{array}{c}j \\
\left(\mathrm{~mA} \mathrm{mg}^{-1}\right)\end{array}$ & $\begin{array}{c}k \\
\left(\mathrm{M}^{-1} \mathrm{~s}^{-1}\right)\end{array}$ & $\begin{array}{c}j \\
\left(\mathrm{~mA} \mathrm{mg}^{-1}\right)\end{array}$ & $\begin{array}{c}k \\
\left(\mathrm{M}^{-1} \mathrm{~s}^{-1}\right)\end{array}$ & $\begin{array}{c}j \\
\left(\mathrm{~mA} \mathrm{mg}^{-1}\right)\end{array}$ & $\begin{array}{c}k \\
\left(\mathrm{M}^{-1} \mathrm{~s}^{-1}\right)\end{array}$ \\
\hline $\mathrm{Pt} / \mathrm{C}$ & 18 & 0,012 & 26 & 0,025 & 20 & 0,013 \\
$\mathrm{Ni}(\mathrm{OH})_{2} / \mathrm{C}$ & 23 & 0,019 & 37 & 0,051 & 20 & 0,015 \\
\hline
\end{tabular}

Uma comparação dos resultados eletrocatalíticos obtidos neste trabalho, através das técnicas de voltametria cíclica e cronoamperometria na oxidação de alcoóis, utilizando eletrodo de carbono vítreo modificado superficialmente por um filme poroso de $\mathrm{Ni}(\mathrm{OH})_{2} / \mathrm{C}$, com alguns trabalhos recentemente publicados utilizando filmes finos de $\mathrm{Ni}(\mathrm{OH})_{2}$ eletrodepositados galvanostaticamente, Kowal e colaboradores, ${ }^{42}$ Rahim e colaboradores ${ }^{43}$ Golikandi e colaboradores,${ }^{44}$ mostra que os resultados estão similares em termos de densidade de corrente (processos faradaicos). Porém, a utilização de filmes eletrodepositados galvanostaticamente, dependendo da espessura dos filmes (tempo de eletrodeposição), é desfavorável economicamente, além do fato destes filmes apresentarem algumas espécies químicas indesejáveis em sua matriz, oriundas dos precursores de níquel e outros surfactantes utilizados. A utilização do $\mathrm{Ni}(\mathrm{OH})_{2} / \mathrm{C}$ torna-se vantajosa, pois apresenta baixíssimo custo de obtenção e o hidróxido é suportado na superfície do carbono vítreo na forma pura.

\section{CONCLUSÕES}

A síntese do $\mathrm{Ni}(\mathrm{OH})_{2} / \mathrm{C}$ se procedeu de forma satisfatória, como comprovado pela técnica DRX, confirmando a estrutura cristalina do respectivo hidróxido sem a existência de possíveis fases secundárias. $\mathrm{O}$ material apresentou estabilidade eletroquímica, sem apresentar variações drásticas de corrente após sucessivas ciclagens de potencial. A atividade eletrocatalítica, verificada por voltametria cíclica, revelou a potencialidade eletroquímica deste hidróxido frente à oxidação dos alcoóis em questão, apresentando resultados superiores ao catalisador comercial $\mathrm{Pt} / \mathrm{C}$ (E-Tek) em todos os parâmetros eletroquímicos avaliados.

Em condições de estado estacionário, $\mathrm{o} \mathrm{Ni}(\mathrm{OH})_{2} / \mathrm{C}$ apresentou desempenho eletroquímico superior a $\mathrm{Pt} / \mathrm{C}$, além de atingir estabilidade faradaica em aproximadamente $50 \mathrm{~s}$. Diante do exposto, e considerando o baixíssimo custo de obtenção do respectivo catalisador, o $\mathrm{Ni}(\mathrm{OH})_{2} / \mathrm{C}$ surge como um material alternativo frente à eletrocatálise da reação de oxidação de alcoóis de baixo peso molecular, ratificando sua utilização como material anódico em meio alcalino.

\section{AGRADECIMENTOS}

Aos revisores do presente manuscrito que, com valiosas contribuições, muito melhoraram o trabalho. À Fundação Araucária, ao MCT-Finep e CNPQ que contribuíram para o desenvolvimento deste projeto. M. R. da Silva agradece ao CNPQ pela bolsa de estudo.

\section{REFERÊNCIAS}

1. Lamy, C.; Lima, A.; Lerhun, V.; Delime, F.; Coutanceau, C.; Léger, J. M.; J. Power Sources 2002, 105, 283.

2. Simões, M.; Baranton, S.; Coutanceau C.; Appl. Catal., B 2010, 93, 354.

3. Friedrich, K. A.; Geyzers, K. P.; Linke, U.; Stimming, U.; Stumper, J.; J. Electroanal. Chem. 1996, 402, 123.

4. Ewe, H.; Justi, E.; Pesditschek, M.; Energy Conversion 1975, 15, 9.

5. Hauffe, W.; Heitbaum, J.; Electrochim. Acta 1978, 23, 299.
6. Santos, E.; Giordano, M. C.; Electrochim. Acta 1985, 30, 871.

7. Lima, R. B.; Paganin, V.; Iwasita, T.; Vielstich, W.; Electrochim. Acta 2003, 49, 85 .

8. Neto, A. O.; Dias, R. R.; Tusi, M. M.; Linardi, M.; Spinacé, E.; J. Power Sources 2007, 166, 87.

9. Tsiakaras, P. E.; J. Power Sources 2007, 171, 107.

10. Watanabe, M.; Motoo, S.; Electroanal. Chem. Interf. Electrochem. 1975, $60,2675$.

11. Li, Y. S.; Zhao, T. S.; Liang, Z. X.; J. Power Sources 2009, 190, 223.

12. Danaee, I.; Jafarian, M.; Forouzandeh, F.; Gobal, F.; Mahjani, M. G.; Int. J. Hydrogen Energy 2008, 33, 4367.

13. Hameed, R. M. A.; El-Khatib, K.M.; Int. J. Hydrogen Energy 2010, 35, 2517.

14. Provazi, K.; Giz, M. J.; Dall'Antonia, L. H.; Torresi, S. I. C.; J. Power Sources 2001, 102, 224.

15. Maruyama, T.; Arai, S.; J. Electrochem. Soc. 1996, 143, 1383.

16. Nikolov, I.; Darkaoui, R.; Zhecheva, E.; Stoyanova, R.; Dimitrov, N.; Vitanov, T.; J. Electroanal. Chem. 1997, 429, 157.

17. Curilazo, R,; Silva, R. M.; Dall'Antonia, L. H.; Semina 2007, $28,93$.

18. Vidotti, M.; Silva, M. R.; Salvador, R. P.; Torresi, S. I. C.; Dall'Antonia, L. H.; Electrochim. Acta 2008, 53, 4030.

19. Vidotti, M.; Torresi, S. I. C.; Kubota, L. T.; Sens. Actuators, B 2008, 135, 245.

20. Vidotti, M.; Greco, C. V.; Ponzio, E. A.; Torresi, S. I. C.; Electrochem. Commun. 2006, 8, 554.

21. Robertson, P. M.; J. Electroanal. Chem. 1980, 111, 97.

22. Taraszewska, J.; Rosonek, G.; J. Electroanal. Chem. 1994, 364, 209

23. Ciszewski, A.; Milczarek, G.; J. Electroanal. Chem. 1996, 413, 137.

24. Revenga-Parra, M.; García, T.; Lorenzo, E.; Pariente, F.; Sens. Actuators, B 2008, 130, 730 .

25. Motheo, A. J.; Tremiliosi-Filho, G.; Gonzalez, E. R.; Kokoh, K. B.; Léger, J. M.; Lamy, C.; J. Appl. Electrochem. 2006, 36, 1035.

26. Amjad, M.; Pletcher, D.; Smith, C.; J. Electrochem. Soc.: Electrochem. Sci. Technol. 1977, 124, 203.

27. El-Shafei, A. A.; J. Electroanal. Chem. 1999, 471, 89.

28. Jafarian, M.; Moghaddam, R. B.; Mahjani, M. G.; Gobal, F.; J. Appl. Electrochem. 2006, 36, 913.

29. Wendt, H.; Götz, M.; Linardi, M.; Quim. Nova 2000, 23, 538.

30. Spinacé, E. V.; Oliveira Neto, A.; Franco, E. G.; Linardi, M.; Gonzalez, E. R.; Quim. Nova 2004, 27, 648.

31. Wendt, H.; Spinacé, E. V.; Oliveira Neto, A.; Linardi, M.; Quim. Nova 2005, 28, 1066.

32. Casas-Cabanas, M.; Rodríguez-Carvajal, J.; Canales-Vázquez, J.; Laligant, Y.; Lacorre, P.; Palacín, M. R.; J. Power Sources 2007, 174, 414.

33. Liu, Z.; Guo, B.; Hong, L.; Lim, T. H.; Electrochem. Commun. 2006, 8, 83.

34. Cullity, B. D.; Stock, S. R.; Elements of X-Ray Diffraction, $3^{\text {rd }}$ ed., Prentice Hall: New Jersey, 2001.

35. Fleischmann, M.; Korinek, K.; Pletcher, D.; J. Electroanal. Chem. 1971, $31,39$.

36. Fleischmann, M.; Korinek, K.; Pletcher, D.; J. Chem. Soc., Perkins Trans. 1972, 2, 1396.

37. Robertson, P. M.; Electrochim. Acta 1977, 22, 203.

38. Kaulen, J.; Schafer, H. J.; Tetrahedron 1982, 38, 3299.

39. Brown, C. J.; Pletcher, D.; Walsh, F. C.; Hammond, J. K.; Robinson, D.; J. Appl. Electrochem. 1994, 24, 95.

40. Chen, Y. L.; Chou, T. C.; J. Appl. Electrochem. 1996, 26, 543.

41. Bard, A. J.; Faulkner, L. R.; Electrochemical Methods. Fundamentals and Applications, $2^{\text {nd }}$ ed., John Wiley \& Sons: New York, 2001.

42. Kowal, A.; Port, S. N.; Nichols, R. J.; Catal. Today 1997, 38, 483.

43. Rahim, M. A. A.; Hameed, R. M. A.; Khalil, M. W.; J. Power Sources 2004, 134, 160.

44. Golikand, A. N.; Shahrokhian, S.; Asgari, M.; Maragheh, M. G.; Irannejad, L.; Khanchi, A.; J. Power Sources 2005, 144, 21. 